Case Report

\title{
Magnet-Retained Facial Prosthesis Combined with Maxillary Obturator
}

\author{
Mahnaz Hatami, ${ }^{1}$ Hamid Badrian, ${ }^{2}$ Siamak Samanipoor, ${ }^{3}$ and Marcelo Coelho Goiato ${ }^{4}$ \\ ${ }^{1}$ Department of Prosthodontics, School of Dentistry, Shahid Sadoughi University of Medical Sciences, \\ Yazd, Iran \\ ${ }^{2}$ Dental Implant Research Center, School of Dentistry, Isfahan, Iran \\ ${ }^{3}$ Department of Endodontics, School of Dentistry, Shahid Bahonar University of Medical Sciences, \\ Kerman, Iran \\ ${ }^{4}$ Faculty of Dentistry of Araçatuba, University of the State of São Paulo, Jose Bonifacio 1193, \\ 16015-050 Araçatuba, SP, Brazil
}

Correspondence should be addressed to Marcelo Coelho Goiato; goiato@foa.unesp.br

Received 28 January 2013; Accepted 25 March 2013

Academic Editors: I. Anic, S. Anil, R. Crespi, M. A. Qudeimat, and M. J. Wahl

Copyright (C) 2013 Mahnaz Hatami et al. This is an open access article distributed under the Creative Commons Attribution License, which permits unrestricted use, distribution, and reproduction in any medium, provided the original work is properly cited.

Prosthetic rehabilitation of the midfacial defects has always perplexed prosthodontists. These defects lead to functional and esthetic deficiencies. The purpose of this clinical case report was the presentation of the prosthetic rehabilitation of an extraoralintraoral defect using two-piece prosthesis magnetically connected. This prosthesis has dramatically improved the patient's speech, mastication, swallowing, and esthetic.

\section{Introduction}

Acquired midfacial defects may affect patients' speech, mastication, quality of life, psychology, and social behavior [1-4]. Midfacial defects are defined as defects in the middle third of the face in horizontal plane that communicate with intraoral maxillary defects. These defects can be classified into two major categories of midline and lateral-midline midfacial defects. Midline midfacial defects include complete or partial involvement of either nose or upper lip that communicate with an intraoral maxillary defect while the lateral midfacial defects include complete or partial involvement of cheek and orbital contents that communicate with an intraoral maxillary defect [5].

Midfacial defect can result from trauma, burns, most tumors of paranasal sinus, palatal epithelium, minor salivary glands [6-8], congenital abnormalities like vascular malformations [6], and some of other lesions like lethal midline granuloma [9] that require partial or radical maxillectomy.
One of the other causes of such defects is mucormycosis that is caused by a fungus of the order Mucorales that is one of the most rapid fatal fungal infections known to man. Rhinocerebral mucormycosis is the most common type, and its extension to the orbit and brain is quite usual. The location of mucormycosis on the palate is rare and of late occurrence [10].

Large midfacial defects are rarely rehabilitated by surgical reconstruction alone. They usually require a facial prosthesis to restore function and esthetic [11].

In addition, an intraoral prosthesis such as an obturator should restore speech and deglutition. Fabrication of a facial prosthesis challenges the artistic ability of prosthodontists. On the other hand, size and weight of facial prostheses endanger the retention of them. This clinical case report describes the prosthodontic rehabilitation of an edentulous patient with a large midfacial (intraoral-extraoral) defect. The main aim of this rehabilitation was to provide the esthetic 


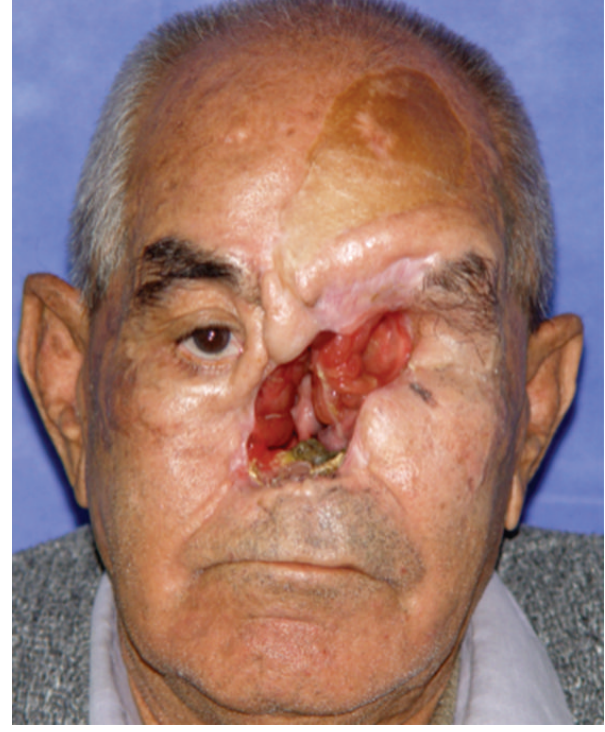

Figure 1: Midfacial defect after surgery.

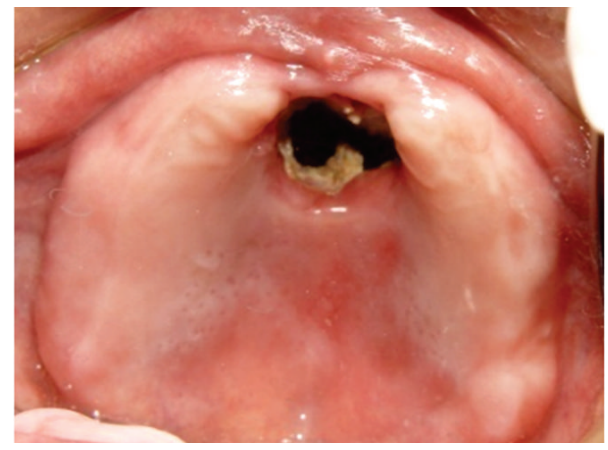

FIGURE 2: Intraoral view of maxillary defect.

needs of the patient and to improve the patient's quality of life.

\section{Case Report}

The patient was a 65 -year-old edentulous male with a chief complaint of poor facial appearance and past medical history of diabetes mellitus. His facial tissues were affected by a fungal infection of rhinocerebral mucormycosis followed by diabetic ketoacidosis. For debridement and removal of necrotic tissues, ablative surgery has been done. Resected portions included anterior part of hard palate, nasal septum and conchae, left maxillary sinus, and orbital contents (Figures 1 and 2). Therefore, there was an open communication between the oral, nasal, and orbital cavities.

After precise evaluation of the case, the proposed treatment plan was to construct a complete denture with obturator, as well as a facial prosthesis which would be attached to the obturator with cobalt samarium magnets (Jobmasters, Randallstown, USA).

Treatment was started by the construction of a complete denture with obturator before facial prosthesis because

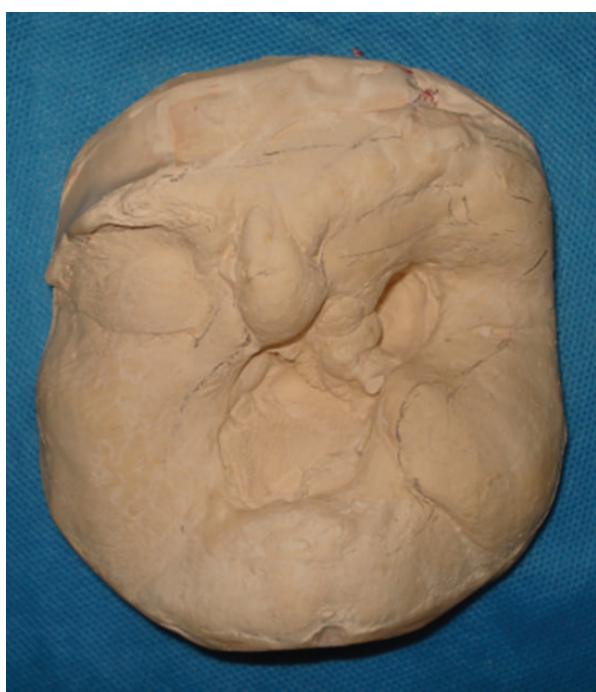

FIgURE 3: Working cast.

anterior-posterior position of anterior denture teeth and labial flange has a basic role in lip support. The complete denture with obturator was made according to the procedure recommended by Zarb et al. [12] and Taylor [13].

The second stage was making an impression of the face defect and adjacent tissues using a thin layer of irreversible hydrocolloid (Hydrogum, Zhermack, Rovigo, Italy). The margin of impression was outlined on the face using the boxing wax (Kerr, Orange, CA, USA). Moist gauze was packed to prevent the flow of impression material into the undesired areas of the defect. The impression was reinforced with fast setting dental plaster (Ernst Hinrichs GMBH, Goslar, Germany) which has a thickness of 0.25 inch. The impression was boxed and poured in dental stone (Ernst Hinrichs GMBH, Goslar, Germany) (Figure 3).

Then working cast was trimmed, defect undercuts were blocked out, and tinfoil substitute was applied to the cast. The waxing up of the nose, orbit, some portions of check and upper lip was developed on a thin acrylic resin baseplate (Acropars, Marlic Co., Tehran, Iran) which adapted on the master cast. Before this step, ocular prosthesis had been made using paper iris disk technique [13]. The cuplike pattern of orbital defect made the basis for inserting the ocular prosthesis within the defect in the same frontal, sagittal, and horizontal planes as the normal eye. The ocular prosthesis was fabricated into a position that matches the gaze of another normal eye when the patient was directly staring at a point at eye level at least 6 feet away. Then eyelid aperture was reproduced by softening and placing two small strips of wax over the ocular section. The shape of the lid opening should make the opening of the other normal eye [13] (Figure 4).

Patient's previous photographs and the references from his first circle relatives were taken as a guide for shaping the wax pattern. The contour of final surface and skin texture was fabricated by carving in lines and wrinkles which observed, by pressing a wet gauze square into softened wax. In the next visit, trial placement of wax pattern was performed on 


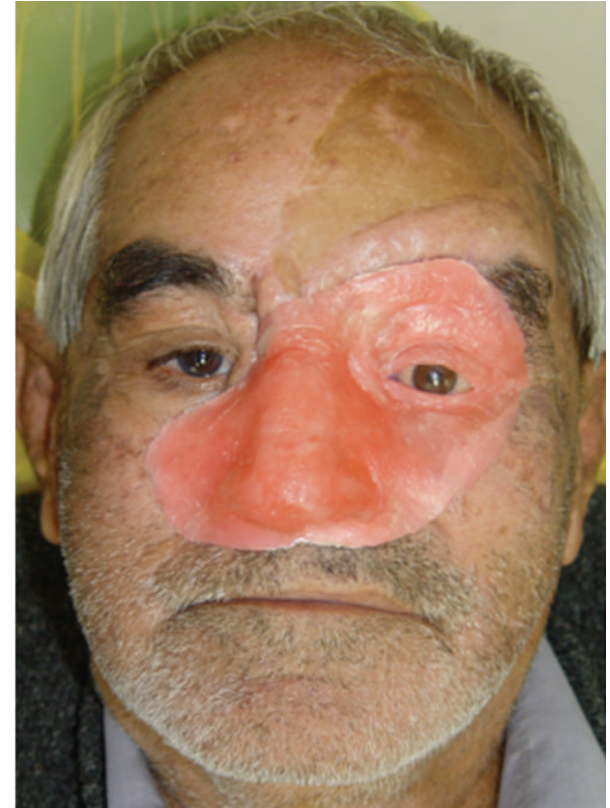

Figure 4: Wax pattern tryin.

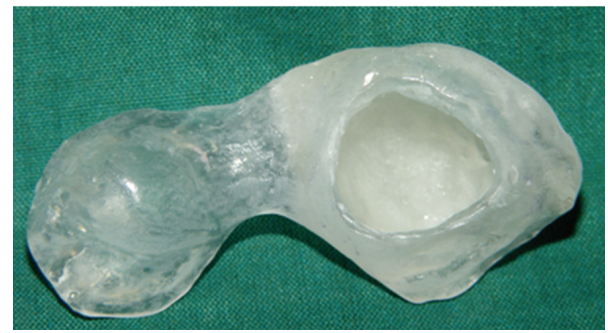

Figure 5: Hollow acrylic substructure.

the patient's face and marginal discrepancies were refitted by corrective wax (Kerr, Orange, CA, USA). Then a hollow acrylic resin framework was made in posterior aspect of wax pattern for decreasing weight of the prosthesis [14] (Figure 5).

The wax pattern was flask using die stone (Ernst Hinrichs GMBH, Goslar, Germany) to form a mold for packing the silicone. Wax elimination was performed in usual manner [15]. Then, the acrylic substructure was placed on the mold and was packed with a MDX4-4210-base silicone (Dow Corning Corp., Midland, USA). Laminar intrinsic staining was used in packing according to the patient's skin color [13]. The silicone was heated for 2 hours at $90^{\circ} \mathrm{C}$, disinvested, trimmed, and cleaned. The prosthesis was trial fitted and extrinsically colored by medical adhesive type A (Dow corning, Midland, MI) and oil pigments (Factor II, Lakeside, USA).

Autopolymerizing resin (Acropars, Marlic Co., Tehran, Iran) was initially used to attach a magnet on the superior aspect of the obturator. Indelible pencil was drawn on the surface of the first magnet, and the facial prosthesis was positioned in its location to demarcate the area of most contact.

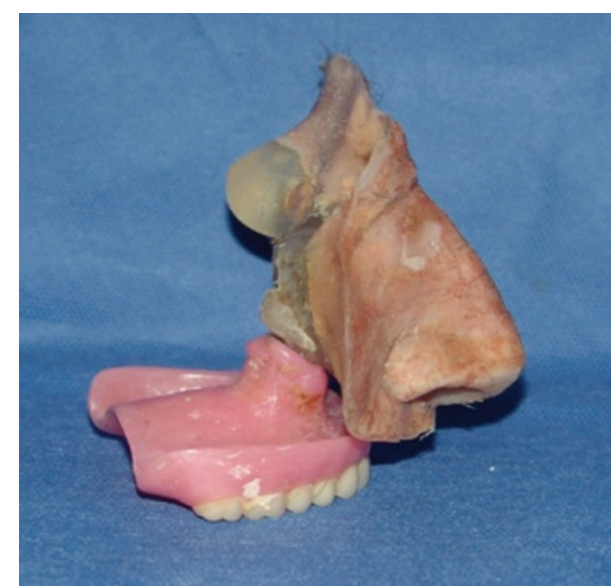

FIGURE 6: Attached intra and extraoral prostheses.

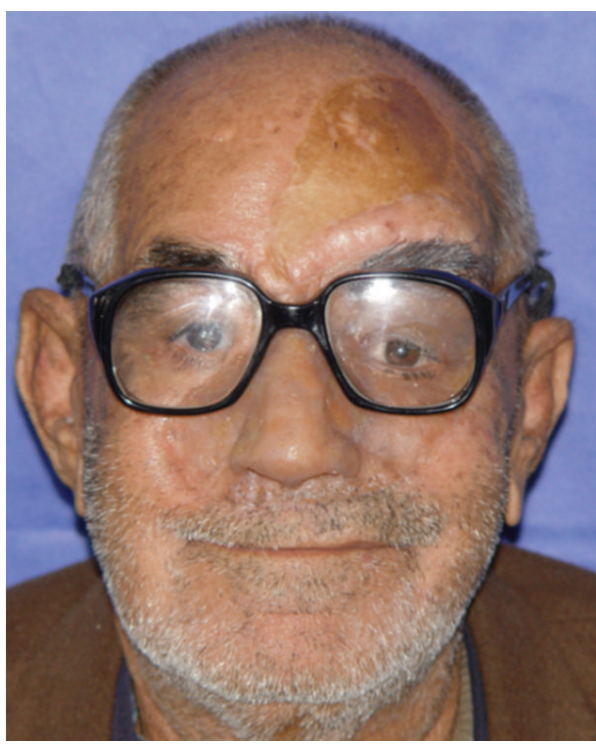

FIGURE 7: Completed facial prosthesis in place.

At the time of insertion of the facial prosthesis, the patient was instructed to close in maximum intercuspal position with obturator placed in the mouth.

On the demarcate area of the facial prosthesis extension (acrylic substructure), the second magnet was attached to self-curing acrylic resin. The extraoral prosthesis had adequate retention after using magnets and eyeglasses (Figures 6 and 7). Extra support for the glasses was gained by attaching an elastic band around the back of the head from one earpiece to another one.

The patient was given hygiene instructions for cleaning both prostheses. The patient attended recall visits every 4 to 5 months. During two years after prosthesis insertion, the prosthesis was still serviceable and the patient was pleased.

An esthetic improvement, intelligible speaking, andimproved deglutition and mastication were achieved for the patient by this prosthetic reconstruction. 


\section{Discussion}

Restoration of midfacial defects can be accomplished surgically or prosthetically or by using a combination of both methods. Selection of each method depends on many factors including size, location of the defect, and age of patient [13].

Acceptable esthetic results usually can be obtained by a facial prosthesis. However, retention of a large prosthesis can be challenging.

Various methods of auxiliary retention for facial prosthesis have been described in the literature; they include eyeglasses [16], denture extensions that engage tissue undercuts $[16,17]$, magnets $[16,18]$, facial prosthetic adhesives [16], or combination of the above $[16,17,19]$, and craniofacial implants $[16,17,20,21]$. Respiratory epithelium is easily traumatized by frictional contact with prosthesis and limits the use of anatomic undercuts [22]. Soft tissues around defects may not always be ideal for adhesive retention because movements that occur during smiling compromise adaptation of prosthesis margins [23, 24].

For the first time, Nadeau [25] described the use of combination of extra- and intraoral prostheses connected by magnets. Durability of surface coatings of the long-term magnets is a major concern; hence, it is advised to use the magnets with strong surface coatings.

Connecting these prostheses often results in movement of facial prosthesis during mastication $[26,27]$. The use of eyeglasses alone for retaining a nasal prosthesis has been well documented [28-30]. Although craniofacial implants may provide the most reliable prosthesis retention, additional surgeries, expenses, inadequate quantity or quality of the bone, and prior radiation to the area may contraindicate this type of treatment $[31,32]$.

A hollow acrylic resin framework used for facial prosthesis is advantageous as there is no need to fabricate the whole prosthesis again in case of discoloration or damage of the silicone layer because the outer silicon layer can be removed and repacked with the new silicon on the acrylic resin framework if the mold is preserved.

The advantages of this prosthesis are that the technique is noninvasive, cost effective, tissue tolerant, esthetic, comfortable to use, and easy to clean. The difficulty in maxillofacial rehabilitation of large defects often involves the compromise of functional adequacy versus esthetic.

\section{Conclusion}

Satisfactory functional and esthetic results are achievable in patients with a large lateral midfacial defect using a hollow acrylic resin framework for silicon facial prosthesis. Retention of facial prosthesis can be satisfactorily achieved with the use of strong magnets provided that the facial prosthesis is light in weight.

\section{Consent}

Informed consent for medical photographs was obtained from the patient.

\section{Conflict of Interests}

The authors report no conflict of interests.

\section{References}

[1] J. B. Ludlow, L. E. Davies-Ludlow, S. L. Brooks, and W. B. Howerton, "Dosimetry of 3 CBCT devices for oral and maxillofacial radiology: CB Mercuray, NewTom 3G and i-CAT," Dentomaxillofacial Radiology, vol. 35, no. 4, pp. 219-226, 2006.

[2] “The glossary of prosthodontic terms," The Journal of Prosthetic Dentistry, vol. 94, no. 1, pp. 10-92, 2005.

[3] C. A. Lascala, J. Panella, and M. M. Marques, "Analysis of the accuracy of linear measurements obtained by cone beam computed tomography (CBCT-NewTom)," Dentomaxillofacial Radiology, vol. 33, no. 5, pp. 291-294, 2004.

[4] D. M. Hecker, J. P. Wiens, T. R. Cowper et al., "Can we assess quality of life in patients with head and neck cancer? A preliminary report from the American Academy of Maxillofacial Prosthetics," Journal of Prosthetic Dentistry, vol. 88, no. 3, pp. 344-351, 2002.

[5] M. T. Marunick, R. Harrison, and J. Beumer, "Prosthodontic rehabilitation of midfacial defects," The Journal of Prosthetic Dentistry, vol. 54, no. 4, pp. 553-560, 1985.

[6] D. M. Hecker, "Maxillofacial rehabilitation of a large facial defect resulting from an arteriovenous malformation utilizing a two-piece prosthesis," Journal of Prosthetic Dentistry, vol. 89, no. 2, pp. 109-113, 2003.

[7] S. Jain, K. Maru, J. Shukla, A. Vyas, R. Pillai, and P. Jain, "Nasal prosthesis rehabilitation: a case report," The Journal of Indian Prosthodontic Society, vol. 11, no. 4, pp. 265-269, 2011.

[8] H. Kurunmäki, R. Kantola, M. M. Hatamleh, D. C. Watts, and P. K. Vallittu, "A fiber-reinforced composite prosthesis restoring a lateral midfacial defect: a clinical report," Journal of Prosthetic Dentistry, vol. 100, no. 5, pp. 348-352, 2008.

[9] S. S. Guttal, N. P. Patil, and A. D. Shetye, "Prosthetic rehabilitation of a midfacial defect resulting from lethal midline granuloma-a clinical report," Journal of Oral Rehabilitation, vol. 33, no. 11, pp. 863-867, 2006.

[10] D. E. Kyrmizakis, P. G. Doxas, J. K. Hajiioannou, and C. E. Papadakis, "Palate ulcer due to mucormycosis," Journal of Laryngology and Otology, vol. 116, no. 2, pp. 146-147, 2002.

[11] R. Brignoni and J. T. Dominici, "An intraoral-extraoral combination prosthesis using an intermediate framework and magnets: a clinical report," Journal of Prosthetic Dentistry, vol. 85, no. 1, pp. 7-11, 2001.

[12] G. A. Zarb, C. L. Bolender, S. E. Eckert, R. Jacob, A. Fenton, and R. Mericske-Stern, Prosthodontic Treatment for Edentulous Patients, Mosby, St. Louis, Mo, USA, 2004.

[13] T. D. Taylor, Clinical Maxillofacial Prosthetics, Quintessence, Carol Stream, Ill, USA, 2000.

[14] P. G. Patil, "Modified technique to fabricate a hollow lightweight facial prosthesis for lateral midfacial defect: a clinical report," The Journal of Advanced Prosthodontics, vol. 2, no. 3, pp. 65-70, 2010.

[15] K. D. Rudd, R. M. Morrow, and J. E. Rhoads, Dental Laboratory Procedures: Fixed Partial Dentures, Mosby, 1986.

[16] K. F. Thomas, J. Penchas, and M. Sela, "Prosthetic rehabilitation," Implant Dentistry, vol. 3, no. 3, p. 198, 1994.

[17] J. B. Ludlow and M. Ivanovic, "Comparative dosimetry of dental CBCT devices and 64-slice CT for oral and maxillofacial 
radiology," Oral Surgery, Oral Medicine, Oral Pathology, Oral Radiology and Endodontology, vol. 106, no. 1, pp. 106-114, 2008.

[18] H. B. Dumbrigue and A. Fylerb, "Minimizing prosthesis movement in a midfacial defect: a clinical report," Journal of Prosthetic Dentistry, vol. 78, no. 4, pp. 341-345, 1997.

[19] Y. Yang, E. Schreibmann, T. Li, C. Wang, and L. Xing, "Evaluation of on-board $\mathrm{kV}$ cone beam CT (CBCT)-based dose calculation," Physics in Medicine and Biology, vol. 52, no. 3, pp. 685-705, 2007.

[20] H. Menneking, M. Klein, B. Hell, and J. Bier, "Prosthetic restoration of nasal defects: indications for two different osseointegrated implant systems," Journal of Facial and Somato Prosthetics, vol. 4, no. 1, pp. 29-33, 1998.

[21] J. Tulasne, P. Worthington, and P. Branemark, Advanced Osseointegration Surgery, Applications in the Maxillofacial Region. Advanced Osseointegration Surgery, Applications in the Maxillofacial Region, 1992.

[22] A. Udagama and G. E. King, "Mechanically retained facial prostheses: helpful or harmful?" The Journal of Prosthetic Dentistry, vol. 49, no. 1, pp. 85-86, 1983.

[23] A. Shifman, "Selective-pressure impression technique for nasal prostheses," The Journal of Prosthetic Dentistry, vol. 58, no. 3, pp. 349-351, 1987.

[24] A. L. Martone, "Anatomy of facial expression and its prosthodontic significance," The Journal of Prosthetic Dentistry, vol. 12, no. 6, pp. 1020-1042, 1962.

[25] J. Nadeau, "Maxillofacial prosthesis with magnetic stabilizers," The Journal of Prosthetic Dentistry, vol. 6, no. 1, pp. 114-119, 1956.

[26] J. Nadeau, "Special prostheses," The Journal of Prosthetic Dentistry, vol. 20, no. 1, pp. 62-76, 1968.

[27] S. Birnbach and G. L. Herman, "Coordinated intraoral and extraoral prostheses in the rehabilitation of the orofacial cancer patient," The Journal of Prosthetic Dentistry, vol. 58, no. 3, pp. 343-348, 1987.

[28] M. F. Gomes, R. Teixeira, G. Plens, M. M. Silva, E. M. Pontes, and J. C. da Rocha, "Naso-orbicular tissue necrosis by Streptococcus parasanguis in a patient with Fanconi anemia: clinical and laboratory aspects," Quintessence International, vol. 35, no. 7, pp. 572-576, 2004.

[29] A. C. Cheng, D. Morrison, and A. G. Wee, "Immediate maxillofacial prosthodontic rehabilitation after radical mid-face tumor resection," Journal of Prosthodontics, vol. 6, no. 4, pp. 265-267, 1997.

[30] S. Rodrigues, V. K. Shenoy, and K. Shenoy, "Prosthetic rehabilitation of a patient after partial rhinectomy: a clinical report," Journal of Prosthetic Dentistry, vol. 93, no. 2, pp. 125-128, 2005.

[31] M. R. Arcuri, W. E. LaVelle, E. Fyler, and R. Jons, "Prosthetic complications of extraoral implants," The Journal of Prosthetic Dentistry, vol. 69, no. 3, pp. 289-292, 1993.

[32] E. Roumanas, R. Nishimura, J. Beumer, P. Moy, J. Weinlander, and J. Lorant, "Craniofacial defects and osseointegrated implants: six-year follow-up report on the success rates of craniofacial implants at UCLA," International Journal of Oral and Maxillofacial Implants, vol. 9, no. 5, pp. 579-585, 1994. 


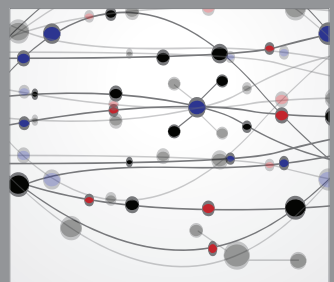

The Scientific World Journal
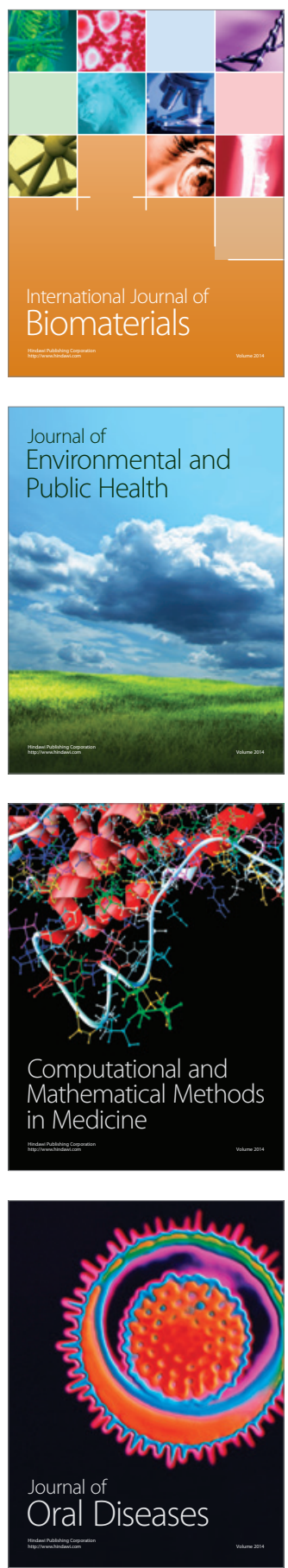
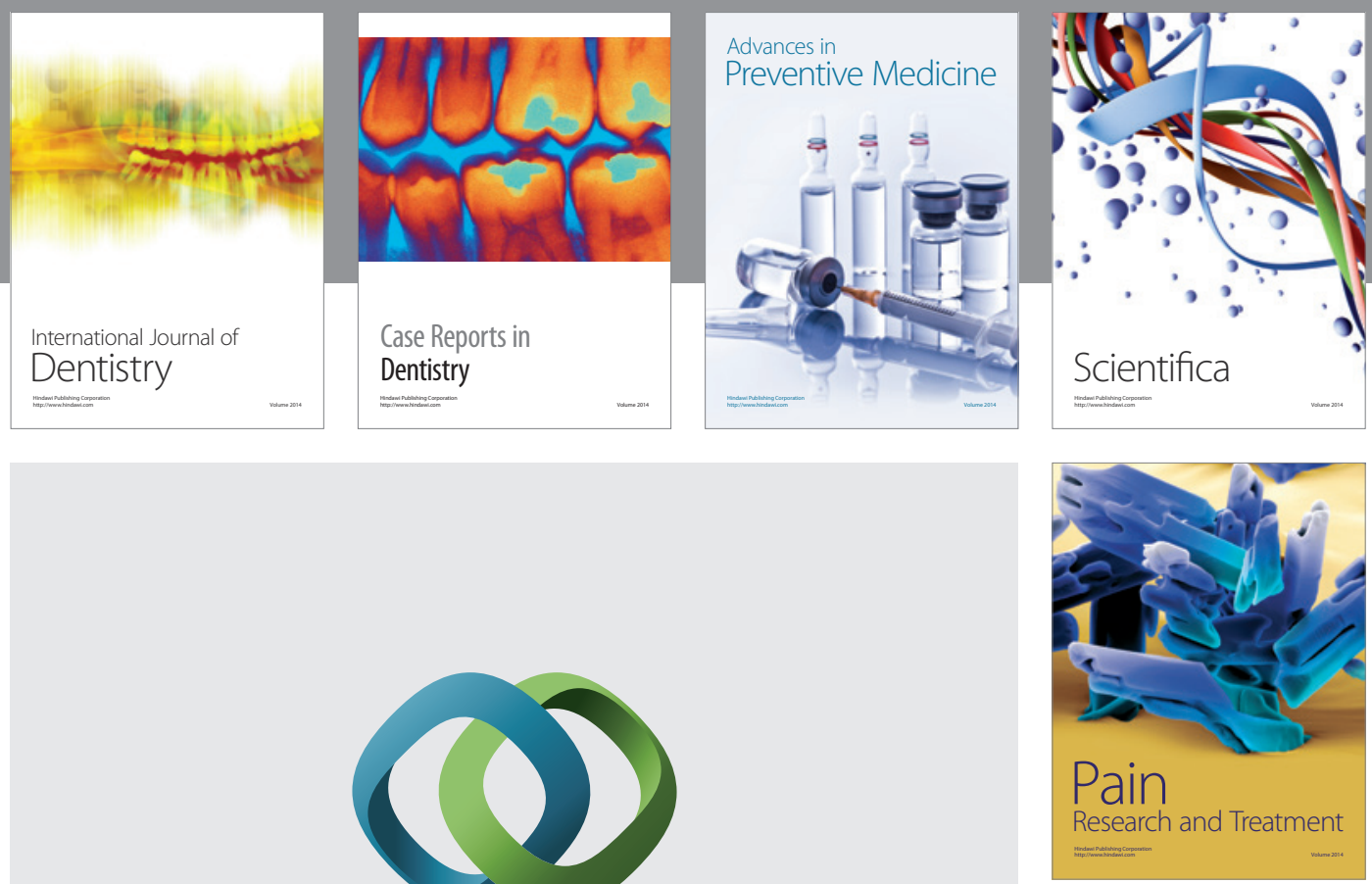

\section{Hindawi}

Submit your manuscripts at

http://www.hindawi.com
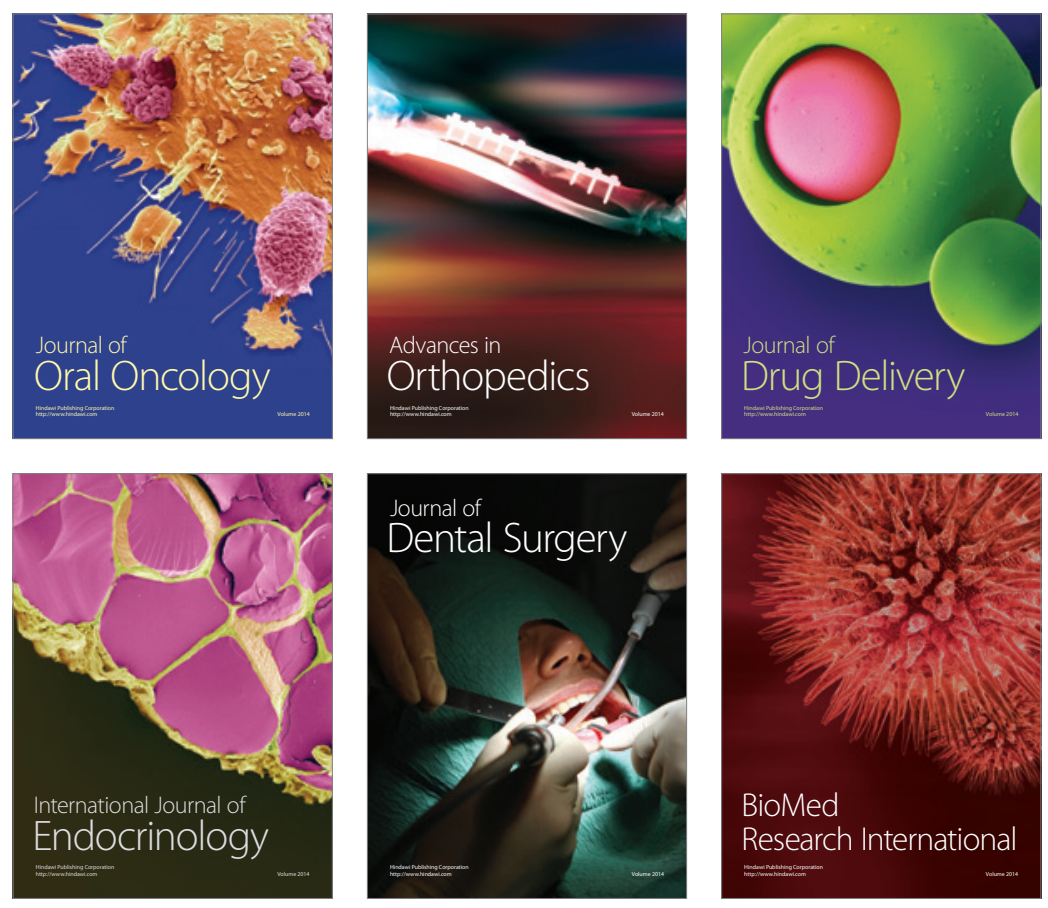

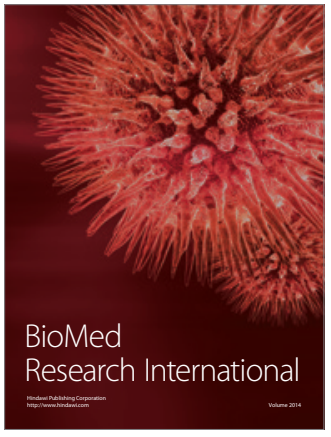

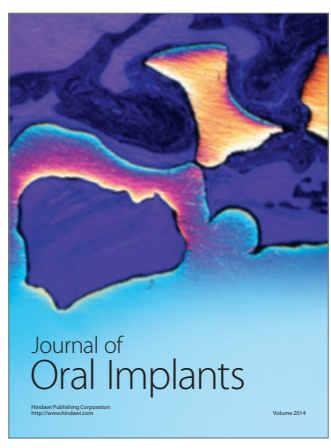
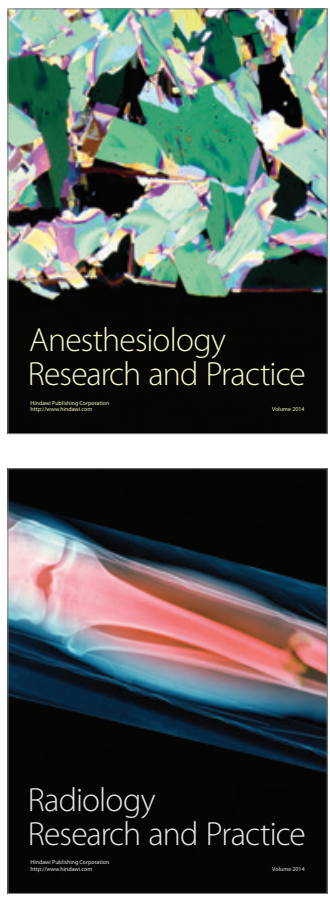